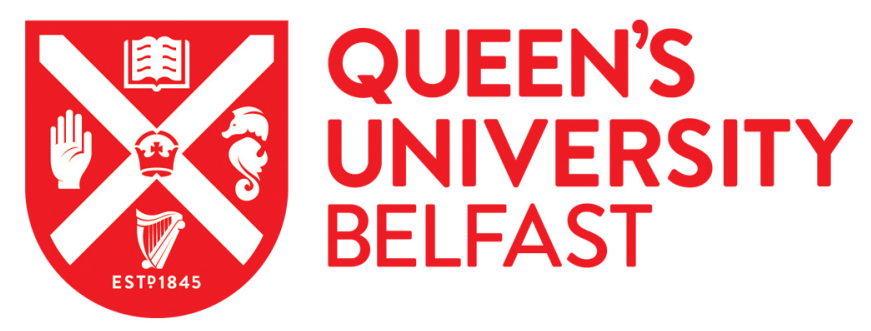

\title{
Pressing Solids Directly Into Sheets of Plasmonic Nanojunctions Enables Solvent-Free Surface-Enhanced Raman Spectroscopy
}

Xu, Y., Ye, Z., Li, C., McCabe, H., Kelly, J., \& Bell, S. E. J. (2018). Pressing Solids Directly Into Sheets of Plasmonic Nanojunctions Enables Solvent-Free Surface-Enhanced Raman Spectroscopy. Applied Materials Today, 13, 352-358. https://doi.org/10.1016/j.apmt.2018.09.014

Published in:

Applied Materials Today

Document Version:

Peer reviewed version

Queen's University Belfast - Research Portal:

Link to publication record in Queen's University Belfast Research Portal

Publisher rights

Copyright 2018 Elsevier.

This manuscript is distributed under a Creative Commons Attribution-NonCommercial-NoDerivs License

(https://creativecommons.org/licenses/by-nc-nd/4.0/), which permits distribution and reproduction for non-commercial purposes, provided the author and source are cited.

\section{General rights}

Copyright for the publications made accessible via the Queen's University Belfast Research Portal is retained by the author(s) and / or other copyright owners and it is a condition of accessing these publications that users recognise and abide by the legal requirements associated with these rights.

Take down policy

The Research Portal is Queen's institutional repository that provides access to Queen's research output. Every effort has been made to ensure that content in the Research Portal does not infringe any person's rights, or applicable UK laws. If you discover content in the Research Portal that you believe breaches copyright or violates any law, please contact openaccess@qub.ac.uk. 


\title{
Accepted Paper: Applied Materials Today
}

\section{Pressing Solids Directly Into Sheets of Plasmonic Nanojunctions Enables Solvent-Free Surface- Enhanced Raman Spectroscopy}

\author{
Yikai Xu, Ziwei Ye, Chunchun Li, Hannah McCabe, Jessica Kelly, Steven E. J. Bell* \\ School of Chemistry \& Chemical Engineering, Queen's University Belfast, BT9 5AG, U.K.
}

Keywords: SERS, solvent-free, nanoparticle sheets, crossing ink lines, co-crystals

\begin{abstract}
Often chemical analysis of solid materials begins with dissolving the sample in a solvent but this is undesirable, particularly if the physical form is important. In principle surfaceenhanced Raman spectroscopy (SERS) should allow detection of solid analytes and offers attomolar sensitivity combined with molecular specificity. SERS requires the target molecules to sit in plasmonic hot-spots, which are normally nanojunctions, just a few nm across. This means that solid samples normally need to be dissolved in a solvent so they can diffuse into the enhancing region. Here, we show that SERS spectra of picograms of solid analytes can be directly obtained by pressing them into a flexible SERS substrate with a dense field of exposed nanojunction hot-spots anchored on its surface. We demonstrate that this can be a powerful tool for straightforward and non-destructive forensic analysis of layered materials (crossing ink lines), solid explosives and illicit drugs as well as for studying previously intractable samples such as pharmaceutical co-crystals, whose important solid state structure is lost when they are dissolved.
\end{abstract}

\section{INTRODUCTION}

Solvents are extensively used throughout analytical science, for example serving as the medium for extracting analytes from a matrix or carrying the sample through analysis [1]. However, the use of solvents also generates additional cost and the possibility of contamination. Moreover, using solvents may not be an option for many real-life applications where the physical form of the sample holds important information or has significant value. Typical examples include analysis of specific crystal forms of therapeutic drugs or forensic evidences. Therefore, there is a strong demand for the development of analytical methods which are solvent-free and nondestructive but at the same time offer high sensitivity. Surface-enhanced Raman spectroscopy (SERS) is a non-destructive and molecular specific analytical technique, which allows routine detection of analytes at micro-gram levels [2-5]. Moreover, by using handheld portable devices 
SERS can be implemented in field for in-situ analysis [6-8]. Here, we show for the first time that SERS enhancements can be readily obtained for a wide range of solid analytes down to picogram levels by forcing bulk crystalline samples into the enhancing hot-spots of robust and flexible nanoparticle-polymer sheets with physical pressure. Importantly, the whole process is solventfree and the pressure required can be achieved manually without any specialist equipment.

The main barrier to solvent-free SERS is that the enhancement arises from the excitation of localized surface plasmons within metallic nanomaterials. This means that the analyte molecules must sit within the overlapping plasmonic hot-spots of closely packed nanostructures, which are typically only a few nanometers across [9-12]. As a result SERS has been largely limited to liquid and gas samples, which can diffuse into enhancing hot-spots. For solid analytes the key challenge is to find a method to bring the sample into the hot-spots without first entering a mobile phase. One possible approach to this problem might be to use tip-enhanced Raman spectroscopy (TERS) type measurements where the enhancing tip is brought close to the solid analyte. This circumvents the geometric problem but comes at the sacrifice of the huge field enhancements which are experienced by molecules located at nanoparticle junctions and requires highly sophisticated equipment $[13,14]$. Similar to the TERS approach, it is also possible to acquire SERS of solid samples by physically bringing them into contact with enhancing materials, such as deposited silver triangles, which have sharp tips $[15,16]$. This avoids the need for specialist TERS equipment but does not provide the nanojunctions which are known to give very high enhancements and have been used in single molecule studies for example [3]. It is possible to obtain SERS signals of highly absorbing compounds such as thiols using solid SERS substrates containing nanojunctions but this relies on the ability of these particular molecules to migrate to the hot spots after adsorption [17-20]. Similarly, SERS signals have been obtained from solid creatine and urea but the sample preparation process included a step which required the analytes to be dissolved in solvent [21]. These methods are not suitable for the vast majority of analytes which are not strongly absorbing or where it may not be desirable or even possible to dissolve the sample.

The method reported here does not place stringent demands on the sample type and so opens a very general route for studying solvent-sensitive analytes with molecular specificity and high sensitivity. Here we show the breadth of applications with examples which range from characterization of pharmaceutical co-crystals to rapid, non-destructive trace analysis of forensic samples, bio-hazards and explosives.

\section{RESULTS and DISCUSSION}

\section{Proof-of-Principle Studies}

The key component is a recently reported new class of flexible SERS substrates, surface exposed nanoparticle sheets (SENS), which are composed of 2-dimensional arrays of NPs physically anchored onto a flexible polymer support (Fig 1a and b) [22]. In these films the nano-gaps are still exposed and accessible, since the particles are only partly submerged into the polymer but the polymer backing makes them physically robust and able to withstand the pressure required to force solid materials into plasmonic enhancing regions to achieve significant signal enhancements (Fig 1b). We have named this new technique surface exposed nanoparticle sheetenhanced Raman spectroscopy (SENSERS). 


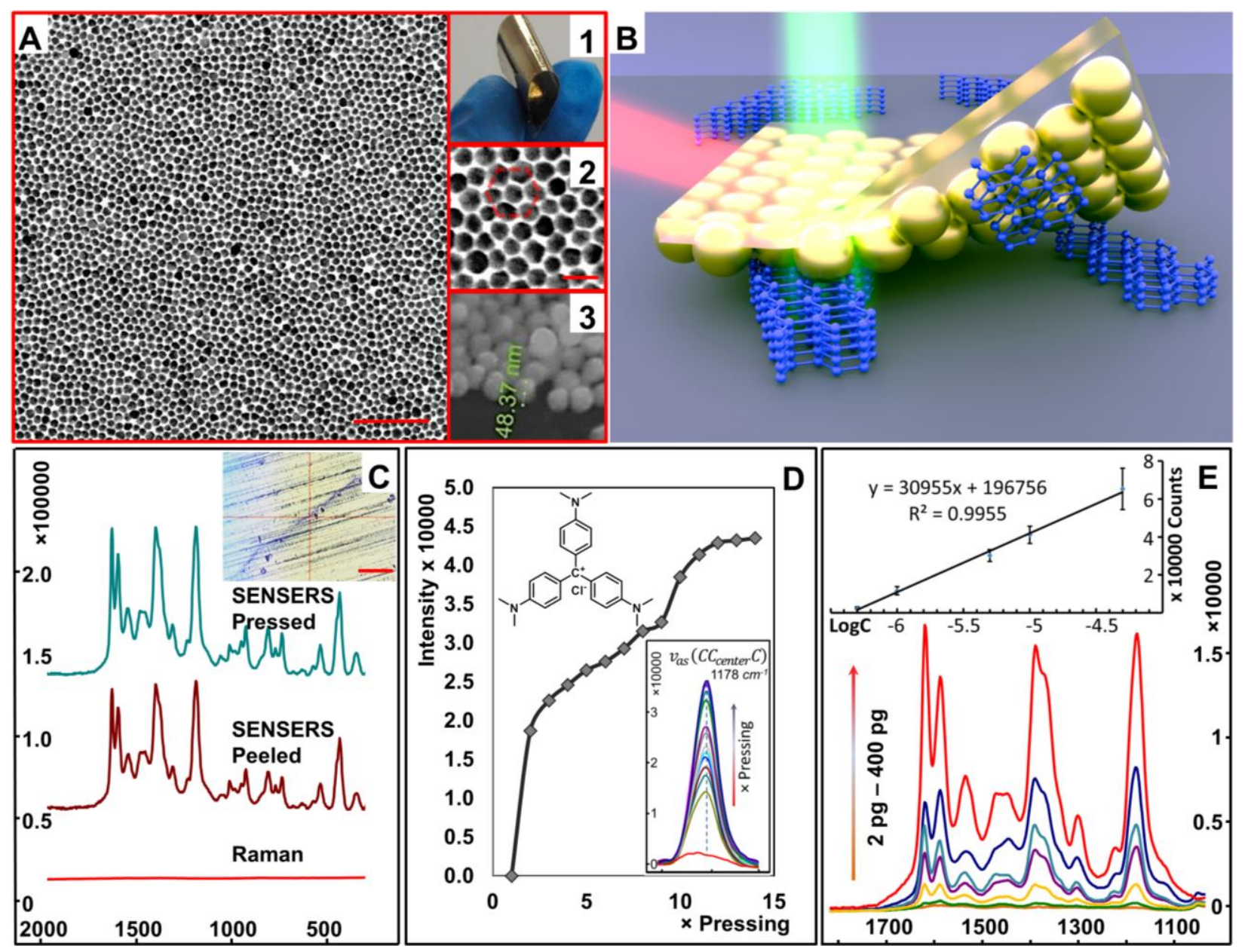

Figure 1. Proof of principle studies using crystal violet (CV). (A) Low magnification scanning electron microscopy (SEM) image of a SENS showing a field of nanoparticles (NPs). Scale bar represents $500 \mathrm{~nm}$. Insets show optical image of SENS (1); high magnification image of the NPs which are packed into a hexagonal lattice with an inter-particle distance measured to be ca. $2 \mathrm{~nm}$. Scale bar represents $100 \mathrm{~nm}$. (2); tilted image showing the NPs anchored in polymer (3). (B) Schematic representation of a surface-exposed nanoparticle sheet enhanced Raman spectroscopy (SENSERS) measurement. (C) Comparing the Raman spectrum of CV coffee ring crystals with SENSERS spectra obtained with the SENS pressed onto the sample and after the SENS was peeled off the sample. Inset shows an optical image of the sample from the Raman microscope. (D) Plot showing the relationship between SERS signal intensity and number of times the sample and SENS were pressed together with a roller (see METHODS SENSERS studies for details). Inset shows the growth of the signal in the SENSERS spectra. (E) SENSERS spectra of CV obtained with different probed sample masses. Inset shows the calibration plot related to the logarithm of $\mathrm{CV}\left(1180 \mathrm{~cm}^{-1}\right.$ peak) from $5 \times 10^{-7}-5 \times 10^{-5} \mathrm{M}$, corresponding to ca. 2$200 \mathrm{pg}$ of probed analyte.

The experimental procedure is very straightforward; firstly since the expected sensitivity of SERS is high, only a small amount of the bulk sample was required, so the samples were typically in the form of thin $(<100 \mu \mathrm{m})$ solid deposits or finely divided powders which were placed on a solid support. The SENS was placed on top of the sample and then pressed onto it 
using moderate pressure ( $80 \mathrm{~N} / \mathrm{cm}^{2}$, see METHODS SENSERS studies for details), forcing the sample into close contact with the SENS. This pressure can be easily achieved manually without any specialist equipment and is well below the pressure $\left(10^{4} \mathrm{~N} / \mathrm{cm}^{2}\right)$ that would induce changes in the crystal or molecular structure of the analyte [23]. Enhanced Raman spectra could then be recorded directly from this support/sample/SENS sandwich structure or the enhancing film could be peeled off from the sample and imaged separately (Fig 1b).

Initial studies were carried out with crystal violet $(\mathrm{CV})$. For experimental convenience the samples were deposited from solution onto a solid metal substrate to form easy to locate solid "coffee rings" (ring diameter ca. $5 \mathrm{~mm}$, deposits ca. $50 \mu \mathrm{m}$ across $\mathrm{x}<1 \mu \mathrm{m}$ deep; see METHODS SENSERS studies and Fig. 2 in ref [24] for details) and probed with a $60 \mu \mathrm{m}$ diameter laser spot to average out local variations in the signal intensity. The thickness of the solid deposits depended on the amount of material deposited, so while drying $10 \mu \mathrm{L}$ of $10^{-4} \mathrm{M}$ solutions created rings with detectable Raman signals $10^{-5} \mathrm{M}$ solutions did not (Fig 1c Raman). However, pressing the SENS onto this sample yielded intense solid SERS signals (Fig 1c SENSERS Pressed) which could be detected directly from the sandwich structure. This obvious contrast in signal intensity demonstrates that these are indeed SERS signals created by bringing the sample into contact with the enhancing substrate. It is worth noting that the signal intensity remained the same even when the substrate was peeled off the bulk crystals (Fig 1c SENSERS Peeled). This suggests that only a sub-set of the analyte crystals i.e. those which have been transferred onto the SENS and into the hot-spot regions between the NPs during the pressing process, are SERS active. Indeed, as shown in Figure 2d, the SERS intensity increased with the number of times the sample was pressed onto the film until a maximum was reached, which was when all the hot-spots had been filled. While CV is well-known to be electrostatically attracted to the surface of NPs in solution, it should be noted that this is not the case in our experiments since CV is introduced as a solid analyte. Conversely, with analytes such as 4-mercaptobenzoic acid, which are known to spontaneously adsorb to Au or Ag nanoparticles through covalent bonding, the near maximum SERS signal was observed immediately the SENS was brought into contact with the solid sample even without any applied pressure (see Fig. 4 in ref [24]), in the same way as was reported in previous "stamping" experiments.

As shown in Figure 1e, the SERS intensity of CV increased with increasing mass of the coffee ring but even with just $2 \mathrm{ng}$ in the total sample corresponding to ca. $2 \mathrm{pg}$ of deposit in the $60 \mu \mathrm{m}$ diameter probed laser spot (see Fig. 3 in ref [24] for calculations), sufficient material could be forced into the enhancing region to give a detectable SERS signal. The inset shows the calibration plot of the linear detection range of CV coffee ring obtained from drying $10 \mu \mathrm{L} 5 \times 10^{-}$ ${ }^{7}-5 \times 10^{-5} \mathrm{M}$ solution (corresponding to ca. 2-200 pg within the probed laser spot). The error bars show the standard deviations obtained by taking 5 random spots on the coffee ring for each analyte concentration, the relative standard deviations ranged from 10-19\%. The deviation in signal intensity can arise from both the non-uniformity of the enhancing substrate and the noneven analyte distribution within the sample. To investigate the uniformity of hot-spots on the SENS substrate, we compared the SERS intensity of an adsorbed thiophenol monolayer between 10 random spots each on two separate SENS samples and obtained relative standard deviations of $11 \%$ and $5 \%$, respectively (see Fig. 1 in ref [24]). This shows that the hot spot non-uniformity is not sufficient to account for the 10-19\% variation observed for the pressed samples and therefore that a significant proportion of the signal variation observed in SENSERS spectra arises from the non-uniformity of the probed sample. This level of reproducibility suggests that 
SENSERS can be applied not only for the identification of solid compounds but also as an effective tool for analyte quantification and mapping of analyte distribution.

\section{Mechanism Studies}

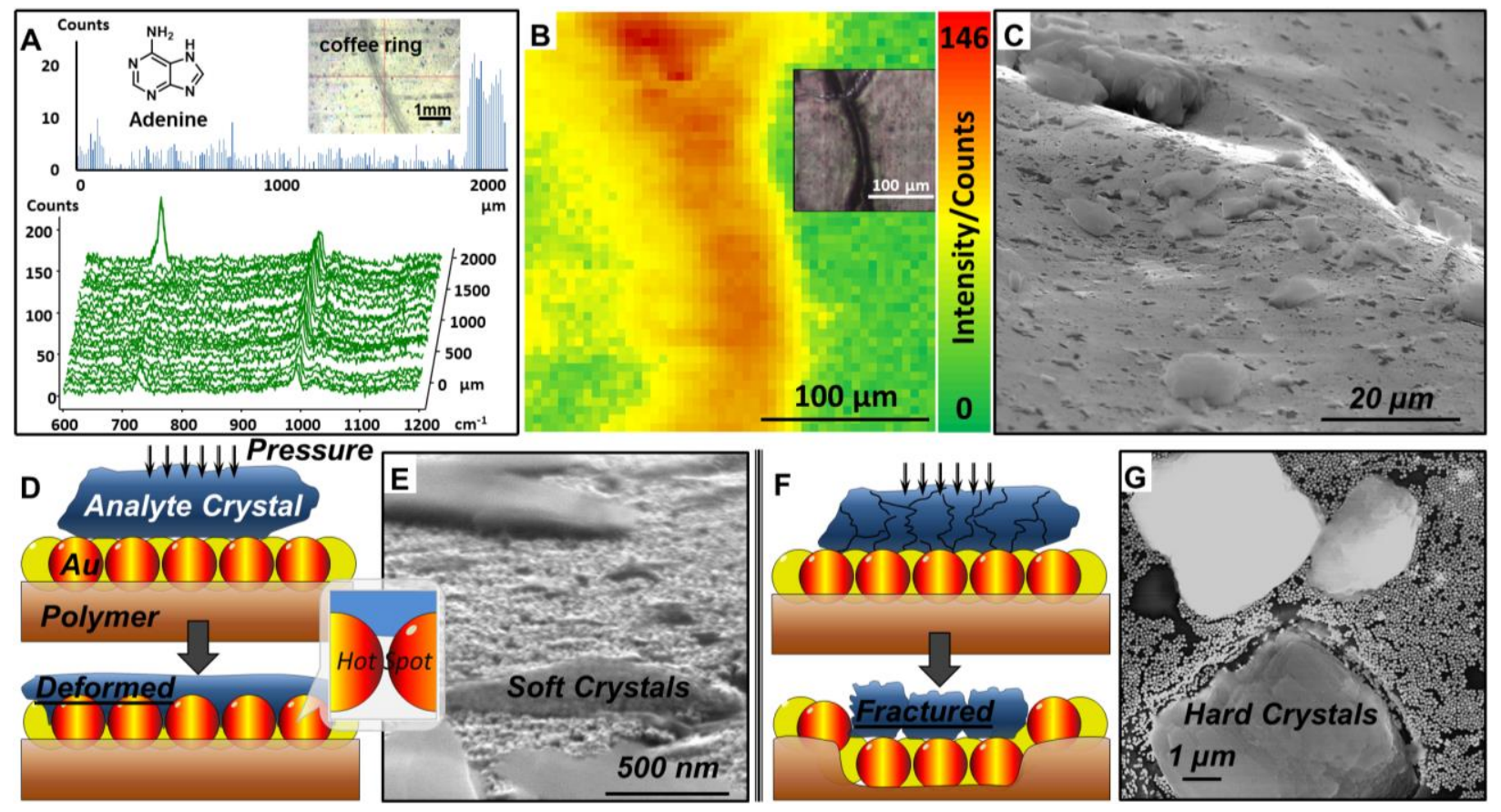

Figure 2. Probing the mechanism of SENSERS with adenine. (A) Mapping the intensity of the $736 \mathrm{~cm}^{-1}$ adenine peak at $10 \mu \mathrm{m}$ intervals from the center of the dried droplet to the edge of the coffee ring deposit using SENSERS shown in bar chart. Below is a subset of the corresponding spectra shown for $20 \mu \mathrm{m}$ spacing. Inset shows the coffee ring with SENS pressed on top imaged under the Raman microscope. (B) SENSERS mapping of a $240 \times 240 \mu \mathrm{m}$ area across the coffee ring moving $5 \mu \mathrm{m}$ per spectrum. Inset shows the optical image of the mapped area. (C) SEM image of the area of SENS that was in contact with the coffee ring crystals. (D) Schematic representations of a soft crystal deforming into hot-spots under pressure to give SENSERS signals. (E) SEM image of thin deformed adenine crystals on the surface of SENS. (F) Schematic representations of a hard crystal fracturing and puncturing the SENS. (G) SEM image of a fragment of a MOF (metal organic framework) crystal puncturing the SENS after pressing.

To further probe the underlining mechanism of SENSERS we have used adenine as the target analyte because it is colorless and therefore avoids complications associated with possible resonance enhancement but gives a well-known characteristic SERS peak at ca. $736 \mathrm{~cm}^{-1}$. Figure 2a shows SENSERS spectra obtained by pressing a SENS film onto a coffee ring deposited from $10 \mu \mathrm{L}$ of $10^{-4} \mathrm{M}$ adenine onto a flat aluminum support. While the detection limit of adenine with SENSERS reaches pg level, for experimental convenience a relatively high concentration was chosen (Fig S1). As shown in Figure 2a, mapping from the center of the dried droplet to the outer edge of the coffee ring gave the largest signals when the probe spot was focused onto the area 
which was in contact with the coffee ring but also that there was reduced but detectable intensity within the area of the dried deposit. This is in good agreement with the expected analyte distribution of a dried coffee ring sample. The consistent peak at $1000 \mathrm{~cm}^{-1}$, which arises from the polystyrene backing [25], shows the uniformity of SENS throughout the mapped area and confirms that the rise and fall of the adenine signal is a result of the analyte distribution. To further investigate SENSERS' ability to reflect the distribution of analyte, Raman mapping of a $240 \times 240 \mu \mathrm{m}^{2}$ area which stretched across a part of the coffee ring was performed. As shown in Figure $2 b$, this gave a SERS intensity map which conforms to the optical image of the sample area observed under the microscope. This further confirmed our earlier observations with CV that the intensity of SENSERS spectra is directly correlated with the amount of sample analyte present and therefore can be used as a sensitive tool for high-resolution SERS mapping of analyte distribution in solid samples. Surprisingly, SENSERS mapping also showed that the signal retained its high intensity even in some regions a significant distance away from the area that the optical image showed was in contact with the coffee ring (Fig 2b and Fig 5 in ref [24]). To investigate this phenomenon further the SENS films were peeled off from the samples and imaged by SEM (scanning electron microscopy). Figure 2c shows the relevant area at low magnification, where the part of the SENS that had contacted the bulk deposit shows clear damage and cratering but away from that area there has also been significant transfer of microcrystalline material during the pressing/peeling process. Some of the debris is in the form of "large" $\left(5 \times 5 \times 5 \mu \mathrm{m}^{3}\right)$ fragments but there are also smaller deposits, which appear as dark patches under normal incidence imaging (see Fig 6 in ref [24]) but with tilted viewing can be seen to be ca. 20-50 nm thick. The e-beam can partly penetrate these thin organic crystals, revealing that the underlying particle array is intact and undamaged (Fig. 2E). Since SERS is a short range effect even these thin deposits are much thicker than would be required to saturate the enhancing surface. Thus, it is expected that SENSERS would be able to detect the presence of solid analytes too thin to be visualized under SEM. Indeed, while clear adenine signals were detected through SENSERS mapping of an area in the middle of a dried adenine sample no observable crystals could be found when the same area of the peeled SENS sample was imaged under the SEM (see Fig 7 in ref [24]).

It is well established in literature that for two $50 \mathrm{~nm}$ diameter gold spheres sitting $2 \mathrm{~nm}$ apart, the $\mathrm{EF}$ (enhancement factor) value reaches a maximum of ca. $10^{8}$ at the point where the two adjacent NPs are closest to each other and decays by orders of magnitude as the inter-particle distance increases [26]. Thus, the level of enhancement will vary for the same analyte at different depths within the inter-particle junction. In the case of SENSERS it is difficult to determine the exact penetration depth of the pressed analytes into the hot-spots. However, even when the analyte is barely pushed into the nanojunction where the distance between the surfaces of adjacent NPs reaches ca. $6 \mathrm{~nm}$, the expected $\mathrm{EF}$ is still at a reasonable value of ca. $10^{5}$. This explains the strong and consistent enhancement observed in SENSERS. Here, we used the "skin" versus "sheet" type analyte distribution model from recent literature to estimate a more precise EF value for SENSERS. In the model, 'skin' type represents liquid or gas analyte samples that can diffuse through gaps to form a uniform analyte layer around the enhancing particles; 'sheet' type represents solid analytes that can only be pressed onto the enhancing surface and into hot-spots following a restricted downward diffusion fashion with an inability to maneuver and penetrate through particularly tight gaps. As a result, 'sheet' type coverage has an EF value up to ca. 3 times lower than 'skin' type coverage [27], and is estimated as ca. $4.5 \times 10^{6}$ for SENSERS (see Fig 8 in ref [24]). This model is also consistent with the observation that SENSERS (sheet type) 
intensity is often weaker but still comparable to the SERS intensity obtained from directly drying the same amount of analyte solution on SENS, which will give skin type coverage (see Fig 9 in ref [24]).

The overall mechanism underlining SENSERS is presented in Figure 2d-g. When pressure is applied to "soft" van der Waals crystals, the crystals deform and fill into the hot-spots in the enhancing particle layer (Fig $2 d$ and e). It should be noted that although the SEM is not able to directly show this, the fact that the particle layers beneath the crystals are undamaged and that strong SERS signals only appear when the sample is pressed hard into the enhancing film is strong evidence that the material has reached the enhancing regions. Conversely, "hard" (i.e. harder than the polystyrene substrate used in the SENS) covalent or ionic crystals such as MOF (metal organic framework) samples fracture into smaller pieces when pressure is applied and may then be pushed through the enhancing particle layer rather than into it (Fig $2 \mathrm{f}$ and $\mathrm{g}$ ). As a result, no enhanced signals can be observed.

\section{SENSERS for Studying Previously Intractable SERS Samples}

Co-crystallizing active pharmaceutical ingredients with biologically safe molecules has recently emerged as a potent way of enhancing the solubility and permeability of drug molecules. Methods to characterize the structures of such artificially engineered crystals are limited by the fact that they dissociate in solvents [28,29]. As such, there have not been any reports on SERS analysis of co-crystal samples since conventional SERS techniques require analytes to be either introduced as a gas or in a liquid medium to allow the target molecules to defuse into hot-spots. SENSERS allow such materials to be directly probed. Figure 3 compares the SENSERS and Raman spectrum of hydrochlorothiazide-nicotinamide (HCT-NCT, 1:1 molar ratio) co-crystals (see Fig S2 for another example). The characteristic peaks of NCT $\left(1047,1654 \mathrm{~cm}^{-1}\right)$ and HCT $\left(1164,1304,1326 \mathrm{~cm}^{-1}\right)$ can be seen in the Raman (3) of the bulk co-crystal. Slight shifts in the positions and relative intensities of the peaks can be seen in the SENSERS spectra (1) and (2). Interestingly, SENSERS spectra of the individual raw materials could only be obtained for NCT but not HCT (Fig S3). This may be due to a change in the hardness of the crystals resulted from the change in crystal structure between pure HCT and its co-crystals. Previously Raman spectroscopy has proved to be an excellent method for distinguishing physical mixtures from cocrystal but this has been limited to investigations of bulk samples [30]. Our results suggest that SENSERS, with its combination of sensitivity and highly localized enhancement, may also provide a route to probe the micro-homogeneity of co-crystal structures. 


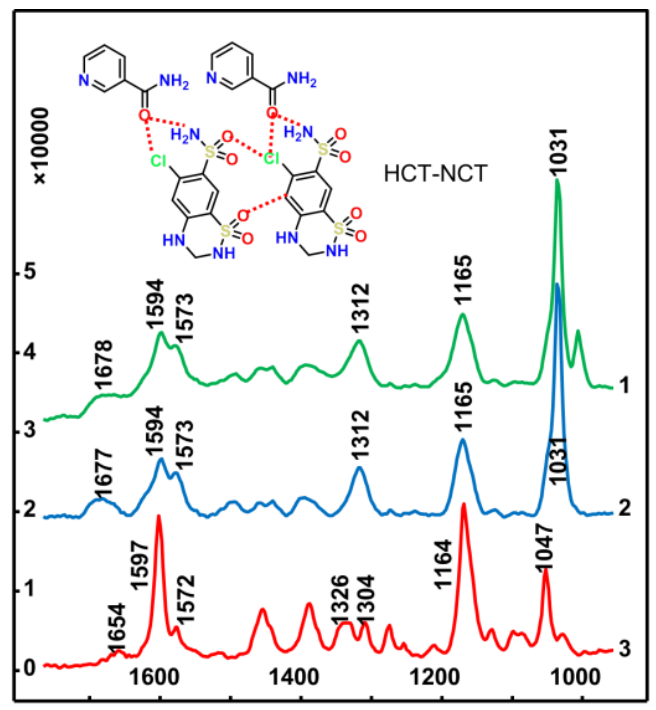

Figure 3. SENSERS for solvent free analysis of artificially engineered co-crystal structures. Unprocessed (1) and background subtracted (2) SENSERS spectra compared with bulk Raman spectra (3) of HCT-NCT. Raman spectra were acquired using ca. $10^{5}$ times more effective sample than SENSERS spectra (see METHODS Instrumentation for details).

\section{SENSERS for Trace Analysis of Real-Life Samples}

Often real-life samples consist of a mixture of unknown compounds that are dispersed in the form of micron dimension powders on topologically complex surfaces. With conventional SERS techniques the crystals must first be extracted from the matrix with solvents before analysis. This dilutes the target, risks sample damage and of course eliminates any information on spatial distribution, which is problematic for precious samples such as forensic evidence or antique artifacts. Here we demonstrate that SENSERS provides a solution to such problems with a variety of non-adsorbing analytes of interest. To mimic real-life samples, $10 \mu \mathrm{L}$ droplets of $5 \times 10^{-5} \mathrm{M}$ solutions were evaporated to create coffee ring crystal structures corresponding to only several hundred pg of analyte within the probed laser spot. Figure 4 shows the SENSER spectra of a commercial explosive (trinitrotoluene, TNT) and an illicit psychoactive drug (3,4methylenedioxymethamphetamine, MDMA i.e. "ecstasy"). Even at these low analyte masses, the characteristic peaks of both molecules can still be clearly observed. It should be noted that both of these molecules have a low affinity for gold and silver NPs. Thus, in conventional SERS studies of MDMA and TNT in solution, substrates with specifically tuned surface properties or sophisticated nanostructures were required [31-33]. Such target specific tuning is not needed in SENSERS since the analytes are physically forced into a large field of enhancing hot-spots, which ensures significant signal enhancement and uniformity. Therefore, SENSERS can be used as a powerful and general technique for non-destructive rapid analysis of real-life samples relevant to homeland security, drug abuse, environmental monitoring etc. 


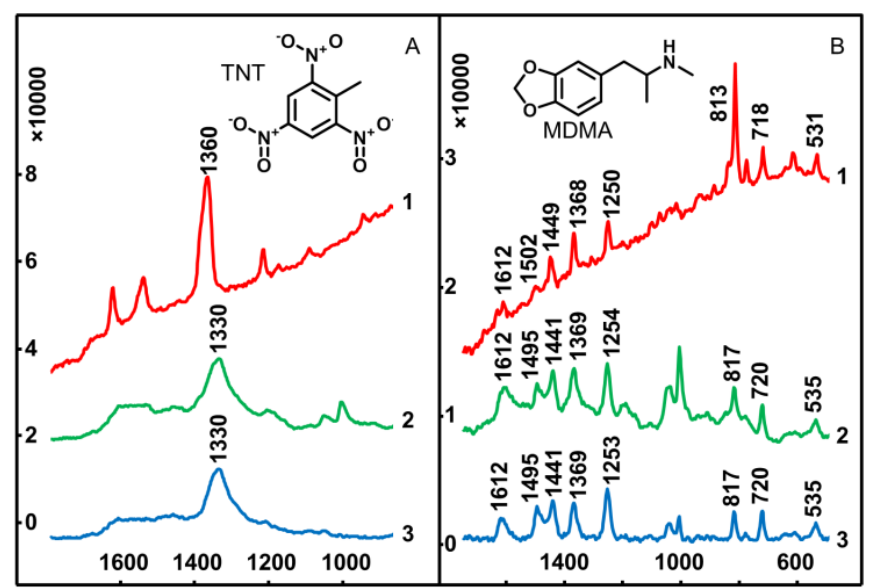

Figure 4. SENSERS for non-adsorbing analytes of real-life interest. (A) Bulk Raman spectra (1) compared with unprocessed (2) and background subtracted (3) SENSERS spectra of TNT. (B) Bulk Raman spectra (1) compared with unprocessed (2) and background subtracted (3) SENSERS spectra of MDMA. Raman spectra were acquired using ca. $10^{5}$ times more effective sample than SENSERS spectra (see METHODS Instrumentation for details).

Finally, we demonstrate that SENSERS can be readily applied to the well-known forensic problem of determining the order of crossing ink lines which commonly arises in cases concerning altered documents. In principle, if a document is altered so that a new ink line is drawn over an existing one the ink molecules of the later will lie on top of the original, so that methods which are depth sensitive should be able to determine the order of the lines. Although various methods based on advanced instrumentations from focused ion beam microscopy to spectrophotometry have been proposed, all have shown limited success [34,35]. As shown in Figure 5 normal Raman measurements of the test sample with crossing blue and green ink lines do not give sufficient discrimination but the SENSERS spectra at the crossing points are indeed dominated by the ink in the uppermost line. The sample shown in the Figure was recorded on a polymer substrate to illustrate that the mechanism of SENSERS means that interfering bands from the sample substrate are not enhanced while the polystyrene band at ca. $1000 \mathrm{~cm}^{-1}$ dominates the normal Raman spectra. Details of the experimental procedure can be found in METHODS SENSERS studies section while corresponding data for a paper substrate is shown in Fig S4.

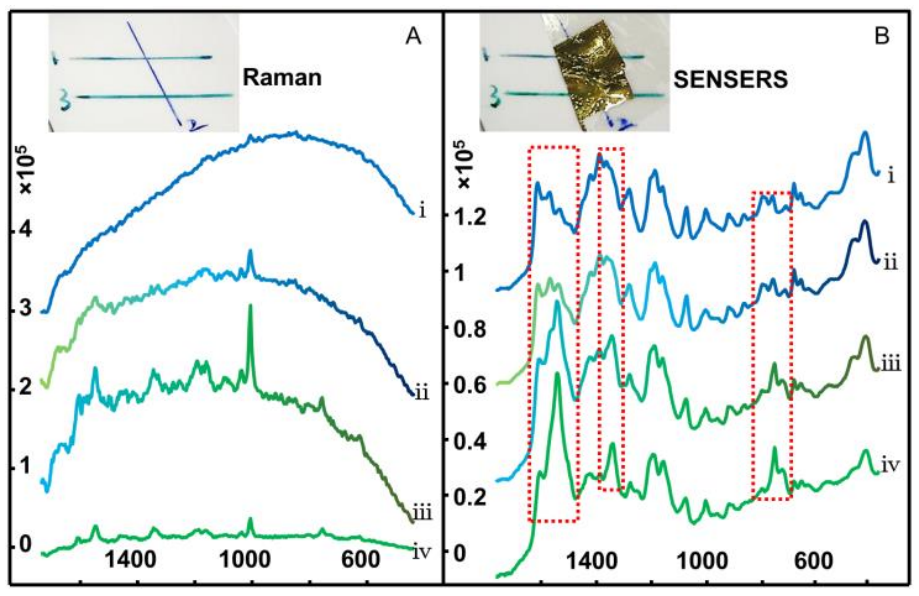


Figure 5. SENSERS for determining the order of crossing ink lines. (A) Raman spectra of different points of the sample. (B) SENSERS spectra of different points of the sample. In both sets of spectra, i-iv corresponds to the spectrum of blue ink, crossing point with blue ink on top, crossing point with green ink on top and green ink, respectively. Insets show images of the sample and the sample with SENS pressed on top, respectively. In inset images 1-3 correspond to the order the lines were drawn (see METHODS SENSERS studies for details).

\section{CONCLUSIONS}

In summary, SENSERS is a new technique for resolving the long-standing limitations of SERS in the direct analysis of solid materials. Since SENSERS is achieved by physically pushing analytes into exposed hot-spots, as opposed to relying on diffusion or adsorption, it is effective for both adsorbing and non-adsorbing analytes and is only limited by the Raman cross-section of the analyte and physical robustness of the SENS. There is clear potential for expanding the approach by developing more robust and flexible polymer substrates and changing the morphology or surface properties of the plasmonic components during fabrication. Since SENSERS uses dry planar substrates it can easily be combined with Raman imaging either at the diffraction limit using standard confocal microscopes or below the diffraction limit using SNOM (scanning near-field optical microscopy) or TERS. Since SENSERS allows SERS measurements of previously inaccessible sample types to be carried out we expect it to find broad application in both fundamental and applied research.

\section{METHODS}

Materials: All chemicals were purchased from Aldrich Ltd. and used without further purification unless otherwise stated. Polystyrene (M. W. ca. 100,000) was purchased from BDH chemicals Ltd. MDMA and TNT were supplied by Forensic Science N.I. Pharmaceutical cocrystals were the kind gifts of Prof. G. Andrews and Ms. C. Lagan (School of Pharmacy, Q.U.B.). CAU-10 MOF samples were the kind gifts of Prof. S. James and Mr. S. Lavery. Water used throughout all experiments was low TOC (<3.0 ppb) $18.2 \mathrm{M} \Omega \mathrm{cm}$ water.

SENS fabrication: Monodisperse $50 \mathrm{~nm}$ diameter gold nano-spheres were prepared following literature methods [36]. The colloid was centrifuged and concentrated $\times 2.5$ before use. SENS were prepared by vigorously shaking $5 \mathrm{~mL}$ of the concentrated aqueous NP colloid with $3 \mathrm{~mL}$ of polystyrene/chloroform $(0.06 \mathrm{~g} / \mathrm{mL})$ solution and $120 \mu \mathrm{L}$ of aqueous tetrabutylammonium nitrate $\left(10^{-3} \mathrm{~mol} / \mathrm{L}\right)$ for ca. $10 \mathrm{~s}$. The agitated mixture was poured immediately into a polypropylene petri dish to form a lustrous metallic film. The mixture was left in air to allow all the dichloromethane to evaporate, which created a robust polymer film ca. $6 \times 3 \mathrm{~cm}^{2}$ in size with a metallic appearance at the water/air interface. The procedure and mechanism is described in full detail in the literature [22,37].

SENSERS studies: Analyte samples were made by either spreading a small amount of analyte crystals onto a rigid metal substrate or drying $10 \mu \mathrm{L}$ of aqueous analyte solution onto the same substrate. For sampling convenience SENS samples were cut with scissors into sections ca. $1 \times 1$ $\mathrm{cm}^{2}$ large and immobilized onto transparent Sellotape ${ }^{\circledR}$ backing with the particle side facing away from the adhesive support to make 'SENSERS stickers'. These stickers were than pressed onto the solid analyte crystals with moderate force $\left(\mathrm{ca} .80 \mathrm{~N} / \mathrm{cm}^{2}\right)$ by rubbing with the fingertips 
or in more controlled manner by pressing with a $30 \mathrm{~mm}$ diameter rigid cylindrical roller. SENSERS spectra could be collected in-situ through the Sellotape and polymer substrate or exsitu from the peeled-off SENS. All spectra shown are averages of ten spectra unless otherwise stated. For studying the relationship between signal intensity and applied pressure, the force with which the SENS was pressed onto the sample with the roller was measured using a digital laboratory balance. Crossing ink line samples were hand-drawn onto paper or polystyrene cardboards with commercial blue and green Sharpie ${ }_{\circledast}$ markers. To mimic altered documents and to ensure no residual solvents were present, each line was dried in the oven at $60{ }^{\circ} \mathrm{C}$ under vacuum for $3 \mathrm{~h}$ before the next line was drawn on top. The sample was directly analyzed under the Raman microscope with the SENS pressed on top. Reference SENSERS spectra of green and blue lines were obtained at points on the ink lines where the two inks did not overlap.

Instrumentation: Conventional optical images were taken with a Nikon COOLPIX L820. Enhanced optical images were taken with an Olympus BX60 microscope fitted with an Olympus digital camera or a Perkin Elmer RamanMicro 200 Raman Microscope. SEM used a Quanta FEG 250 at an accelerating voltage of $30 \mathrm{kV}$ under high chamber pressure $\left(8 \times 10^{-5} \mathrm{mbar}\right)$ with standard SEM copper tape or carbon tape as background. SENSERS spectra were collected with a Perkin Elmer RamanMicro 200 Raman Microscope equipped with a $785 \mathrm{~nm}$ diode laser (60 $\mu \mathrm{m}$ spot diameter at sample) and a $\times 10$ object lens using a total accumulation time of $50 \mathrm{~s}$ and $100 \mathrm{~mW}$ laser power. Normal Raman spectra were collected using ca. $10^{-3} \mathrm{~g}$ of sample, a total of $50 \mathrm{~s}$ accumulation time and $400 \mathrm{~mW}$ laser power. SENSERS mapping was performed with a HORIBA LabRam HR Raman microscope equipped with a $785 \mathrm{~nm}$ diode laser $(2 \mu \mathrm{m}$ spot diameter at sample) using a total accumulation time of $4 \mathrm{~s}$ and $100 \mathrm{~mW}$ laser power. All spectra were further analyzed with Grams AI software (Thermofisher Inc.).

\section{ASSOCIATED CONTENT}

Supporting Information includes: substrate uniformity characterizations; SENSERS data of adsorbing analytes; calculations of EF; calculations of effective analyte mass; detail SEM characterizations; additional Raman mapping; additional SENSERS data; This material is available free of charge via the Internet.

\section{AUTHOR INFORMATION}

Corresponding Author

*Correspondence: s.bell@qub.ac.uk

Funding Sources

Yikai Xu was funded by the University Special Research Scholarship (Q. U. B.).

Notes

The authors declare no competing financial interests

\section{ACKNOWLEDGMENT}


The author would like to thank Professor AP de Silva for his invaluable advice on the manuscript; Lei Gao and Chengxi Guo for their help with the figures; Prof. G. Andrews, Ms. C. Lagan, Prof. S. James, Mr. S. Lavery and Prof. T. E. Keyes for their experimental support.

\section{REFERENCES}

1. Z. Zhang, M. J. Yang, J. Pawliszyn, Solid-phase microextraction, Anal. Chem. 66 (1994) 844-853.

2. M. P. Cecchini, V. A. Turek, J. Paget, A. A. Kornyshev, J. B. Edel, Self-assembled nanoparticle arrays for multiphase trace analyte detection, Nat. Mater. 12 (2013) 165171.

3. S. Nie, S. R. Emory, Probing single molecules and single nanoparticles by surfaceenhanced Raman scattering, Science 275 (1997) 1102-1106.

4. S. Dick, M. P. Konrad, W. W. Y. Lee, H. McCabe, J. N. McCracken, T. M. D. Rahman, A. Stewart, Y. Xu, S. E. J. Bell, Surface-enhanced Raman spectroscopy as a probe of the surface chemistry of nanostructured materials, Adv. Mater. 28 (2016) 5705-5711.

5. Y. C. Cao, R. Jin, C. A. Mirkin, Nanoparticles with Raman spectroscopic fingerprints for DNA and RNA detection, Science 297 (2002) 1536-1540.

6. N. Chen, P. Ding, Y. Shi, T. Jin, Y. Su, H. Wang, Y. He, Portable and reliable surfaceenhanced Raman scattering silicon chip for signal-on detection of trace trinitrotoluene explosives in real systems, Anal. Chem. 89 (2017) 5072-5078.

7. R. Pilot, SERS detection of food contaminants by means of portable Raman instruments, J. Raman Spectrosc. 49 (2018) 954-981. 
8. D. W. Li, W. L. Zhai, Y. T. Li, Y. T. Long, Recent progress in surface enhanced Raman spectroscopy for the detection of environmental pollutants, Microchim. Acta 181 (2014) $23-43$.

9. K. A. Willets, R. P. van Duyne, Localized surface plasmon resonance spectroscopy and sensing,Annu. Rev. Phys. Chem. 58 (2007) 267-297.

10. J. Theiss, P. Pavaskar, P. M. Echternach, R. E. Muller, S. B. Cronin, Plasmonic nanoparticle arrays with nanometer separation for high-performance SERS substrates, Nano Lett. 10 (2010) 2749-2754.

11. N. J. Halas, S. Lal, W. S. Chang, S. Link, P. Nordlander, Plasmons in strongly coupled metallic nanostructures, Chem. Rev. 111 (2011) 3913-3961.

12. M. Tebbe, S. Lentz, L. Guerrini, A. Fery, R. A. Alvarez-Puebla, N. Pazos-Perez, Fabrication and optical enhancing properties of discrete supercrystals, Nanoscale 8, (2016) 12702-12709.

13. J. F. Li, Y. F. Huang, Y. Ding, Z. L. Yang, S. B. Li, X. S. Zhou, F. R. Fan, Z. Wei, Z. Y. Zhou, D. Y. Wu, B. Ren, Z. L. Wang, Z. Q. Tian, Shell-isolated nanoparticle-enhanced Raman spectroscopy, Nature 464 (2010) 392-395.

14. R. Zhang, Y. Zhang, Z. C. Dong, S. Jiang, C. Zhang, L. G. Chen, L. Zhang, Y. Liao, J. Aizpurua, Y. Luo, J. L. Yang, J. G. Hou, Chemical mapping of a single molecule by plasmon-enhanced Raman scattering, Nature 498 (2013) 82-86. 
15. T. Liyanage, A. Rael, S. Shaffer, S. Zaidi, J. V. Goodpaster, R. Sardar, Fabrication of a self-assembled and flexible SERS nanosensor for explosive detection at part-perquadrillion levels from fingerprints, Analyst 143 (2018) 2012-2022.

16. X. Wang, X. Zhu, Y. Chen, M. Zheng, Q. Xiang, Z. Tang, G. Zhang, H. Duan, Sensitive surface-enhanced Raman scattering detection using on-demand postassembled particleon-film structure, ACS Appl. Mater. Interfaces 9 (2017) 31102-31110.

17. Y. Chen, K. J. Si, D. Sikdar, Y. Tang, M. Premaratne, W. Cheng, Ultrathin plasmene nanosheets as soft and surface-attachable SERS substrates with high signal uniformity, Adv. Optical Mater. 3 (2015) 919-924.

18. T. Wadayama, M. Oishi, A. Hatta, Surface enhanced Raman scattering of organic sample powders spread over vacuum-evaporated silver thin films, Appl. Surf. Sci. 253 (2006) 2713-2717.

19. P. Wang, L. Wu, Z. Lu, Q. Li, W. Yin, F. Ding, H. Han, Gecko-inspired nanotentacle surface-enhanced Raman spectroscopy substrate for sampling and reliable detection of pesticide residues in fruits and vegetables, Anal. Chem. 89, (2017) 2424-2431.

20. J. Chen, Y. Huang, P. Kannan, L. Zhang, Z. Lin, J. Zhang, T. Chen, L. Guo, Flexible and adhesive surface enhanced Raman scattering active tape for rapid detection of pesticide residues in fruits and vegetables, Anal. Chem. 88 (2016) 2149-2155.

21. M. Li, Y. Du, F. Zhao, J. Zeng, C. Mohan, W. C. Shih, Reagent- and separation-free measurements of urine creatinine concentration using stamping surface enhanced Raman scattering (S-SERS), Biomed. Opt. Express 6 (2015) 849-858. 
22. Y. Xu, M. P. Konrad, J. L.Trotter, C. P. McCoy, S. E. J. Bell, Rapid one-pot preparation of large freestanding nanoparticle-polymer films, Small 13 (2017) 1602163.

23. E. V. Boldyreva, High-pressure-induced structural changes in molecular crystals preserving the space group symmetry: anisotropic distortion/isosymmetric polymorphism, Cryst. Eng. 6 (2003) 235-254.

24. C. Li, Z. Ye, H. McCabe, J. Kelly, Y. Xu, S. E. J. Bell, Dataset demonstrating the working-principle of surface-exposed nanoparticle sheet enhanced Raman spectroscopy, Data in Brief, submitted.

25. J. R. Anema, A. G. Brolo, A. Felten, C. Bittencourt, Surface-enhanced Raman scattering from polystyrene on gold clusters, J. Raman Spectrosc. 41 (2010) 745-751.

26. P. G. Etchegoin, E. C. Le Ru, A perspective on single molecule SERS: current status and future challenges, Phys. Chem. Chem. Phys. 10 (2008) 6079-6089.

27. D. M. Solís, J. M. Taboada, F. Obelleiro, L. M. Liz-Marzán, Optimization of nanoparticle-based SERS substrates through large-scale realistic simulations, ACS Photonics 4 (2017) 329-337.

28. Ö. Almarsson, E. B. Vadas, Molecules, materials, medicines (M3): linking molecules to medicines through pharmaceutical materials science, Cryst. Growth Des. 15 (2015) 56455647.

29. N. K. Duggirala, M. L. Perry, Ö. Almarsson, M. J. Zaworotko, Pharmaceutical cocrystals: along the path to improved medicines, Chem. Comm. 52 (2016) 640-655. 
30. J. C. Burley, A. Alkhalil, M. Bloomfield, P. Matousek, Transmission Raman spectroscopy for quality control in model cocrystal tablets, Analyst 137 (2012) 30523057.

31. A. Stewart, S. E. J. Bell, Modification of Ag nanoparticles with mixed thiols for improved SERS detection of poorly adsorbing target molecules: detection of MDMA, Chem. Comm. 47 (2011) 4523-4525.

32. S. R. D. Samuel, A. K. Singh, D. Senapati, H. Yu, P. C. Ray, Gold nanoparticle based label-free SERS probe for ultrasensitive and selective detection of trinitrotoluene, J. Am. Chem. Soc. 131 (2009) 13806-13812.

33. X. He, H. Wang, Z. Li, D. Chen, J. Liu, Q. Zhang, Ultrasensitive SERS detection of trinitrotoluene through capillarity-constructed reversible hot spots based on $\mathrm{ZnO}-\mathrm{Ag}$ nanorod hybrids, Nanoscale 7 (2015) 8619-8626.

34. K. Bojko, C. Roux, B. J.Reedy, An examination of the sequence of intersecting lines using attenuated total reflectance-fourier transform infrared spectral imaging, J. Forensic Sci. 53 (2008) 1458-1467.

35. A. Braz, M. López-López, C. García-Ruiz, Raman imaging for determining the sequence of blue pen ink crossings, Forensic Sci. Int. 249 (2015) 92-100.

36. N. G. Bastús, J. Comenge, V. Puntes, Kinetically controlled seeded growth synthesis of citrate-stabilized gold nanoparticles of up to $200 \mathrm{~nm}$ : size focusing versus Ostwald ripening, Langmuir 27 (2011) 11098-11105. 
37. Y. Xu, M. P. Konrad, W. W. Y. Lee, Z. Ye, S. E. J. Bell, A method for promoting assembly of metallic and nonmetallic nanoparticles into interfacial monolayer films, Nano Lett. 16 (2016) 5255-5260.

\section{DATA AVAILABILITY}

The raw/processed data required to reproduce these findings cannot be shared at this time as the data also forms part of an ongoing study.

Table of Contents Graphic

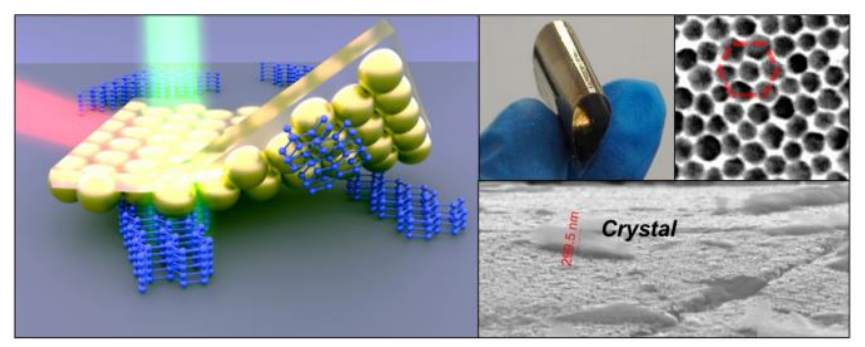

